\title{
The microbiome as a therapy in pouchitis and ulcerative colitis
}

Jonathan P Segal ${ }^{1}$, Jean-Frédéric LeBlanc ${ }^{2}$, Lucia Maria de Campos Braz ${ }^{2,3}$, Ailsa L Hart ${ }^{2,3}$

1. Department of Gastroenterology, The Hillingdon Hospital, Uxbridge, United Kingdom

2. Inflammatory Bowel Disease Unit, St. Mark's Hospital, Harrow, United Kingdom

3. Faculty of Medicine, Department of Metabolism, Digestion and Reproduction, Imperial College, London, United Kingdom

Jonathan P Segal $(1,2)$ BSc (Hons) MBChB, PhD Orcid ID : 0000-0002-9668-0316

Jean-Frédéric LeBlanc MD, FRCPC Orcid ID : 0000-0002-8477-6337

Lucia Maria de Campos Braz Bsc 0000-0002-9572-7717

Ailsa L Hart BA(Hons), BMBCh, FRCP, PhD (1,2) Orcid ID: 0000-0002-7141-6076

Author for Correspondence:

Jonathan Segal

Hillingdon Hospital

Pield Heath Road

UB8 3NN

Jonathansegal1@nhs.net

Main text word count: 4751

References: 68

Tables and figures: 0:2

Disclosures: none of the authors have any relevant disclosures.

Guarantor of the Article: Ailsa Hart

Author Contributions: JPS, JFL, LMB conducted the literature review and prepared the manuscript. AH provided supervision of the manuscript and gave critical revisions. All authors have agreed on the final version. 
Key Words: Ulcerative colitis, Ileoanal pouch Inflammatory bowel disease, Microbiota

\begin{abstract}
:
The gut microbiome is important in the homeostasis of gut health and has pivotal roles in digestion, immune regulation, and metabolic processes. The gut microbiome has been implicated in range of diseases and there is a rapidly growing understanding of this ecosystem's importance in inflammatory bowel disease. We have yet to identify a single microbe that causes either ulcerative colitis or pouchitis, however, perturbations in the gut microbiome are associated with disease states. Importantly, we can manipulate the gut microbiome using dietary interventions, medications, and faecal microbiota transplantation. This review will summarise our knowledge of gut microbiome therapies in ulcerative colitis and pouchitis.
\end{abstract}




\subsection{Introduction}

Our human gut microbiome is composed of a vast range of microorganisms that include, bacteria, virus, archaea, and protozoa, that together form an extremely complex ecosystem capable of communicating with our immune system and determining our predisposition to develop disease states. The human gut microbiome is responsible for a range of metabolic processes for the host including digesting food specially through the breakdown of complex carbohydrates and proteins, regulation of the immune function, protecting against pathogens and production of micronutrients ${ }^{1}$.

Our microbiome composition is determined by a number of host and environmental factors to include mode of delivery, type of feeding at birth, use of antibiotics, pathogen exposure, diet, smoking, pollutants as well as many unknown factors ${ }^{2}$.

Ulcerative colitis is a relapsing and remitting inflammatory bowel disease which is increasing in incidence and prevalence. It currently affects 10 per 100,000 people annually, with a prevalence of 243 per 100,000 . This amounts to approximately 146,000 patients in the UK with a diagnosis of ulcerative colitis ${ }^{3}$.

When trying to understand the role of the gut microbiome on the aetiology of ulcerative colitis, longitudinal data are lacking. A particular challenge remains that we still are unable to predict those that will develop ulcerative colitis and therefore we lack the ability to map the microbiome prior to disease onset.

In an attempt to circumnavigate this issue, a model for ulcerative colitis may provide us unique insights into the role of the gut microbiome in inflammatory bowel disease. In such a model, the key will be to explore microbiome changes from a period of health into a disease state, mapping the microbiome changes. Ideally these changes need to occur over a period where longitudinal data is possible minimising loss to follow-up. 
The ileoanal pouch offers this unique opportunity for a variety of reasons. Firstly, an ileoanal pouch is formed in those with ulcerative colitis who remain refractory to medical therapy and hence have a genetic predisposition to inflammation. Secondly, the ileoanal pouch has a high incidence of inflammation at one year called pouchitis, allowing longitudinal data in a short timeframe. Lastly, the mainstay of treatment for pouchitis remains antibiotics and many studies have implicated the importance of the microbiome in both aetiology and treatment.

This review aims to summarise the potential role of the microbiome as a therapeutic target in both ulcerative colitis and pouchitis.

\subsection{Uncovering microbiome-based therapies through mechanistic understanding of pathogenesis in ulcerative colitis}

Through advancing next generation sequencing technologies we have been able to understand both the composition of the gut microbiome but also functionality ${ }^{4}$. Although data remains heterogenous, a consistent finding of perturbations in the gut microbiome in patients with UC compared with healthy controls persist.

The gut microbiome seems an integral part in maintaining tight junction integrity ${ }^{5}$. Evidence has suggested that perturbations in the gut microbiome can lead to an increase in gut permeability, a decrease in the thickness of the protective mucus layer which culminates in pathogen invasion ${ }^{6}$. The goblet cells that produce a thick mucus layer harbour microbiota that may have a protective role. Therefore, a loss of this mucus layer as found in gut inflammation leads to a loss in mucosal integrity and impaired barrier function. This culminates in an increase in bacterial translocation altering the $T$ cell profile which begin to circulate pro-inflammatory cytokines leading to tissue damage, a destabilized microbiome and further tissue damage. 
From an immune perspective the gut microbiome is in constant communication with the immune system to help with immune tolerance ${ }^{7}$ and disease pathogenesis. There is much evidence that the gut microbiome can directly communicate to the immune system which indirectly influences its direction dependent on the metabolites produced (Figure 1).

Figure 1. The role of the gut microbiome

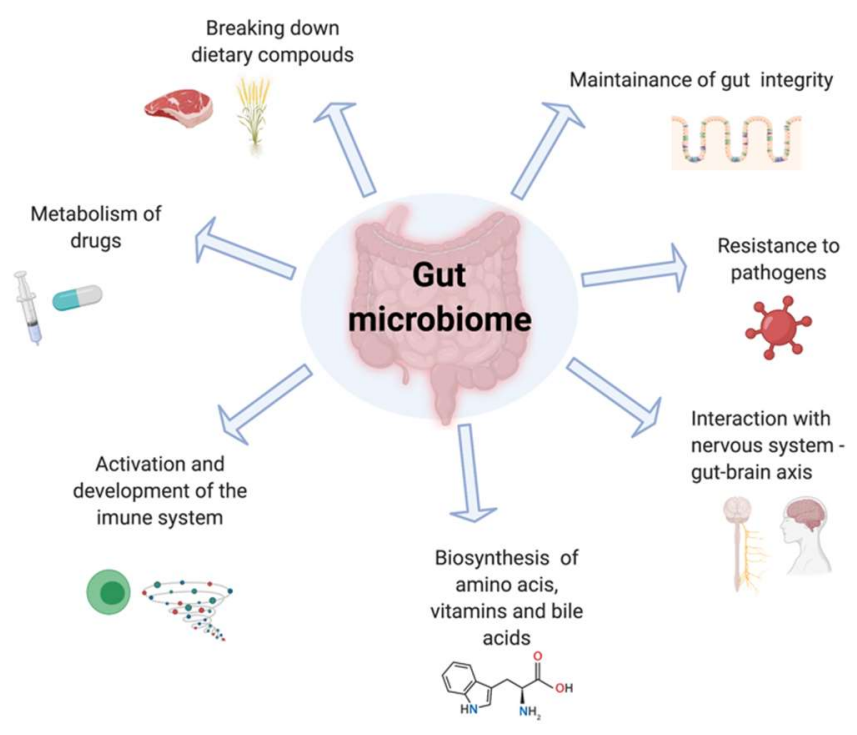

1.2 Uncovering microbiome-based therapies through mechanistic understanding of pathogenesis in pouchitis

The microbiota of a patient with pouchitis differs from a patient without pouchitis ${ }^{8}$. Specific patterns have found persistence of Fusobacter and Enteric species associated with the disease state and the absence of specific bacteria such as Streptococcus species in the inflamed pouch ${ }^{9}$. Clinically, pouchitis can be treated with antibiotics and hence provides plausibility that the microbiome may influence the course of pouchitis. 
Of interest, mucosal inflammation in the pouch is concentrated in areas where bacterial concentration is highest ${ }^{10}$. From an immune perspective, it has been noted that pouchitis-derived bacterial sonicates from metronidazole-sensitive bacterial species stimulate healthy patients' mononuclear cells significantly more than corresponding sonicates from non-pouchitis patients.

Potentially, the closest direct link to the microbiome having an influence on pouchitis came from a study which highlighted that baseline microbiome prior to colectomy could predict those that developed pouchitis and those that did not ${ }^{11}$ and hence provides suggestions that altering the gut microbiome may influence the pouch functionality.

\section{$\underline{\text { 2.1 Prebiotic studies in Ulcerative colitis }}$}

The gut microbiome can be manipulated through the use of prebiotics, probiotics, antibiotics and faecal microbiota transplantation and diet (Figure 2).

Figure 2. Methods of manipulating the gut microbiome 


\section{IBD microbiome}

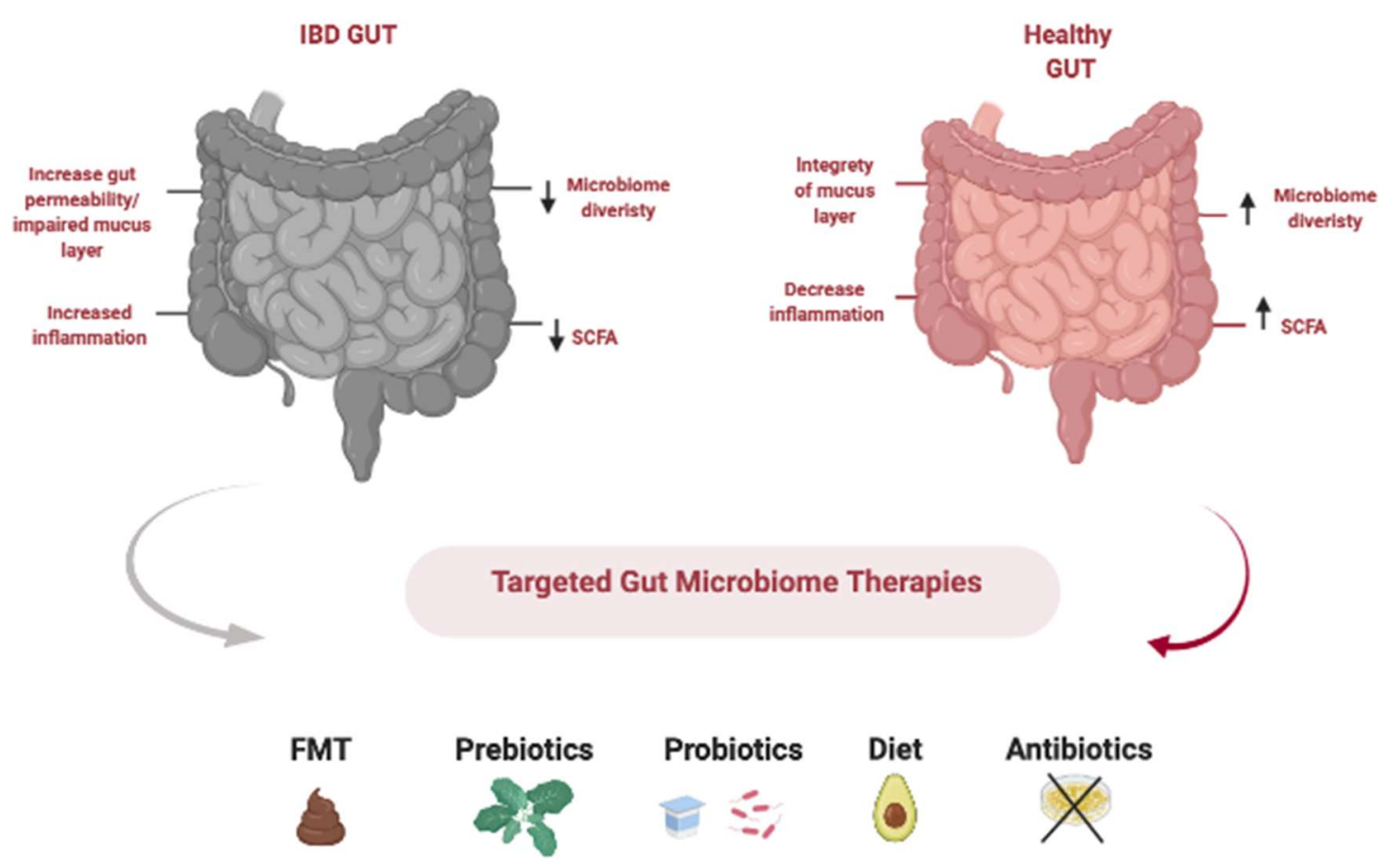

Dietary prebiotics are defined as "a substrate that is selectively utilised by host microorganisms conferring a health benefit"12. It is hypothesised that prebiotics may improve gut inflammation by selective stimulation of protective members of gut microbiota, improvement of the intestinal permeability and increased production of short chain fatty acids (SCFA) ${ }^{13}$.

Hafer et al. studied the effect of lactulose at a daily dose of $10 \mathrm{~g}$ added to standard therapy in 7 patients with active UC, compared to 7 UC patients receiving standard therapy without the use of a placebo. They noted that the Inflammatory Bowel Disease Questionnaire (IBDQ) score improved from $123 \pm 20$ to $171 \pm 18(p=0.026)$ in the lactulose group ${ }^{14}$.

Two clinical trials evaluated the effects of an oligofructose-enriched inulin compound (Beneo ${ }^{\mathrm{TM}}$ Synergy 1) in UC patients with mild to moderate disease, receiving concomitant mesalazine therapy. Casellas et al. noted that, at day 14 , levels of faecal 
calprotectin improved in five of seven patients (70\%) who received the prebiotic, compared to two of eight patients $(25 \%)$ in the placebo arm ${ }^{15}$.

Valcheva et al. assessed the alterations of the gut microbiota composition and activity in 25 patients with mild to moderate UC treated with different doses of oligofructoseenriched inulin over a nine-week period. The primary outcome was clinical response and/or remission. The primary outcome was achieved in $77 \%$ of patients receiving the high-dose prebiotic product (15 g per day) compared to 33\% in the low-dose group (7.5 g per day). High-fructan dose was associated with Bifidobacteriaceae and Lachnospiraceae abundance, however such microbiota modifications did not correlate with improved disease scores. Interestingly, the trial showed that a prebiotic course resulted in higher butyrate levels, with strong negative correlations between butyrate levels and clinical symptoms ${ }^{16}$. Existing trials assessing the use of prebiotics in the treatment of UC lack sufficient power to change clinical practice, however data regarding its potential efficacy and safety profile are encouraging.

\subsection{Probiotic studies in Ulcerative colitis}

Probiotics are "live microorganisms which confer a health benefit on the host when administered in adequate amounts" 12 . Probiotics are traditionally composed of one or more bacterial strains.

Derwa et al. performed a meta-analysis of eight trials targeting induction of remission in active UC as a primary outcome $(n=651)$, as well as six trials assessing prevention of relapse in quiescent UC $(n=677)^{17}$. Types of probiotics varied between $E$. coli Nissle 1917 (5 studies), Bifidobacterium longum 356 (1 study), Lactobacillus rhamnosus GG (1 study), a multistrain probiotic containing a combination of lactic acid bacteria, streptococci and bifidobacteria(3 studies) and other combined formulations (4 studies). In the single trial comparing probiotics with 5-ASAs for induction of remission, no difference was seen in the primary outcome of failure to achieve remission $(R R=1.24$; $95 \% \mathrm{Cl}=0.70-2.22$ ), similar to the pooled 7 placebo-controlled $\mathrm{RCTs}(\mathrm{RR}=0.86 ; 95 \%$ 
$\mathrm{Cl}=0.68-1.08)$. Rates of adverse events were comparable in both analyses. Interestingly, in a subgroup analysis of the multistrain probiotic containing a combination of lactic acid bacteria, streptococci and bifidobacteria studies, $56.2 \%$ of 162 patients randomised to the probiotic failed to achieve remission, compared with $75.2 \%$ of 157 patients who received placebo $(\mathrm{RR}=0.74 ; 95 \% \mathrm{Cl}=0.63-0.87)$. The authors measured a number needed to treat of 5 to prevent one patient with active $U C$ failing to achieve remission, without significant heterogeneity between studies $\left(\mathrm{I}^{2}=0 \%, \mathrm{P}=0.52\right)$. The $E$. coli Nissle 1917 compound did not demonstrate a statistically different benefit compared to placebo ( $\mathrm{RR}=1.56 ; 95 \% \mathrm{Cl}=0.44-5.53)$. In regard to maintenance of remission, probiotics were not shown to decrease rates of UC relapse compared with 5-ASAs $(\mathrm{RR}=1.02 ; 95 \% \mathrm{Cl}=0.85-1.23)$ and with placebo $(\mathrm{RR}=0.62 ; 95 \% \mathrm{Cl}=0.33-1.16)$.

Astó et al. conducted a meta-analysis of RCTs examining the effects of probiotics, prebiotics and synbiotics on human $\mathrm{UC}^{18}$. Rates of remission in patients with active UC were unchanged between the probiotics and placebo groups. In trials defining UC remission, the beneficial effects of probiotics were estimated to be statistically significant compared to placebo $(\mathrm{RR}=1.55,95 \% \mathrm{Cl}=1.13-2.15)$ with decreased heterogeneity between trials $\left(I^{2}=29 \%\right)$. On further subgroup analysis $(n=424)$, patients with active UC who received Bifidobacterium-containing probiotics were more likely to be in remission compared to those on placebo $(R R=1.73 ; 95 \% \mathrm{Cl}=1.23-2.43, \mathrm{P}=0.002)$. In comparison, no difference in UC remission was seen between probiotics without Bifidobacterium strains and control groups $(n=168)$. In trials assessing the multistrain probiotic containing a combination of lactic acid bacteria, streptococci and bifidobacteria in combination with standard therapy $(n=348)$, significantly higher rates of $U C$ remission were seen in the probiotic group compared to the control group $(R R=1.99 ; 95 \%$ $\mathrm{Cl}=1.25-3.15, \mathrm{P}=0.003)$.

The faecal concentrations of SCFA were measured in 2 trials and were significantly increased in one pilot study with active UC patients after supplementation of Bifidobacterium-fermented milk $(n=20)^{19}$. In a trial assessing inactive UC patients, SCFA concentrations did not differ significantly between probiotic (Streptococcus faecalis $\mathrm{T}$ 110, Clostridium butyricum TO-A and Bacillus mesentericus TO-A) and placebo groups 
at any time over the six months, however a higher butyrate/acetate ratio was observed throughout the follow-up period in patients who relapsed compared to those who remained in remission ${ }^{20}$. Decreased Bifidobacterium species was observed in inactive UC patients prior to relapse in one study assessing the effects of Bifidobacterium breve fermented milk ${ }^{21}$. Furrie et al. explored the effects of a synbiotic formulation containing B. longum and oligofructose-enriched inulin; improved endoscopic scores and significantly higher levels of bifidobacterial rRNA on mucosal biopsies were observed in patients receiving this synbiotic compared to those on placebo ${ }^{22}$.

\section{$\underline{\text { 2.3 Antibiotics in Ulcerative colitis }}$}

Antibiotics are seldom used in clinical practice for the management of UC. Khan et al. performed a meta-analysis of nine randomised clinical trials assessing the efficacy of antibiotics in adult patients with active UC ${ }^{23}$. Efficacy outcomes were mostly clinical with limited reporting of biochemical and endoscopic outcomes. Overall, the authors noted a statistically significant benefit favouring antibiotics over placebo $(\mathrm{RR} 0.64 ; 95 \% \mathrm{Cl}=$ 0.43-0.96, $\mathrm{P}=0.03$ ), however antibiotic regimens were significantly heterogeneous between trials. Internationally-recognised guidelines either do not recommend their use or do not mention them as a potential therapeutic option in the management of adult patients with $U C^{24,25}$.

\subsection{Faecal Transplant in Ulcerative colitis}

Since 2015, four placebo-controlled RCTs ${ }^{26-29}$ and multiple cohort studies have been published $^{30-32}$, with meta-analyses suggesting a positive impact of FMT in the induction of remission in UC patients with mild-moderate disease ${ }^{33}$.

Costello et al. conducted a meta-analysis of the four-placebo controlled RCTs. Clinical remission was achieved in $28 \%$ of pooled donor FMT groups compared with $9 \%$ of 
patients in placebo groups $(\mathrm{OR}=3.67 ; 95 \% \mathrm{Cl}=1.82-7.39)^{34}$. A Danish open-label pilot study has examined the efficacy of oral FMT capsules in patients with active UC; over a 50-day course of oral FMT capsules, all of the seven patients achieved clinical response at weeks 4 and 8 , as well as significant improvements in quality of life and faecal calprotectin levels ${ }^{30}$.

Paramsothy et al. performed gastrointestinal microbial community profiling in UC patients treated with colonoscopy delivered FMT versus placebo. Bacterial diversity in samples before and after FMT administration was higher in recipients who achieved remission compared with those who did not. Remission after FMT was associated with a relative microbial abundance of Eubacterium hallii and Roseburia inulivorans, compared with higher levels of Fusobacterium gonidiaformans, Sutterella wadsworthensis and Escherichia species in patients not determined to be in remission post-FMT. The former patient group exhibited increased production of SCFA and secondary bile acids, while the latter group showed higher levels of heme and lipopolysaccharide biosynthesis. A relative abundance of Bacteroides species in donor FMT stools was associated with higher rates of remission in recipients, likely explained by antagonist interactions between Bacteroides and Prevotella species ${ }^{35}$.

The beneficial effects of FMT in UC may also be mediated by other members of the intestinal microbiota, such as viruses and fungi. In a small cohort of UC patients, a numerical trend towards reduction in eukaryotic viral richness was observed in FMT responders compared with non-responders $(p=0.056)^{36}$. The pro-inflammatory role of Candida species was highlighted in the Leonardi et al. trial. A relative abundance of Candida species pre-FMT was associated with increased bacterial diversity, which likely implies a microbiota more amenable to FMT engraftment. A reduction of Candida species post-FMT administration correlated with improved clinical and endoscopic outcomes; such an impact was not reproduced in patients receiving placebo. ${ }^{37} \mathrm{~A}$ better understanding of the FMT-induced modifications of bacterial taxonomy, as well as transkingdom interactions, will hopefully improve the selection process of FMT donors and recipients, thus improving the overall efficacy of FMT in the management of active UC. 


\subsection{Dietary studies in UC}

In one of the largest studies exploring diet in Inflammatory bowel disease which included 413593 participants from 8 European countries, the EPIC-IBD (European Prospective Investigation into Cancer and Nutrition - Inflammatory Bowel Diseases) cohort aimed to investigate the relationship between diet and IBD. They reported that vegetable protein intake was not associated with IBD risk. However, there was an association between meat consumption and disease (adjusted HR for the fourth vs. the first quartile $=1.37 ; \mathrm{Cl} 195 \%=1.02-1.82, P$-trend $=0.003)$ and between red meat consumption and IBD risk (adjusted HR for the fourth vs. the first quartile $=1.41 ; \mathrm{Cl} 95 \%$ $=1.03-1.92, P$-trend $=0.006)^{38}$. There have been other studies highlighting the importance of macronutrients in the aetiology of $\mathrm{IBD}^{39}$ with limited data regarding their impact on the gut microbiome. In one study that explored the role of animal-based diets, it was found that the increase in the abundance and activity of Bilophila wadsworthia on the animal-based diet group supports a link between dietary fat and the overgrowth of microbes able to prompt the host to IBD ${ }^{40}$.

\section{$\underline{2.6 \text { Specific Ulcerative colitis diets }}$}

A diet low in FODMAP (fermentable oligosaccharides, disaccharides, monosaccharides and polyols) may likely improve the quality of life of patients with irritable bowel syndrome (IBS) ${ }^{41}$. Data is scant regarding the effects of a diet low in FODMAPs in the clinical improvement of patients with IBD. An RCT was performed to investigate the effects of a low FODMAP diet on persistent gut symptoms, microbiome diversity and markers of inflammation in patients with quiescent IBD ${ }^{42}$. Patients following a low FODMAP diet $(14 / 27,52 \%)$ reported improved gut symptoms 4 weeks after following the dietary regime, compared with control diet $(4 / 25,16 \%, P=.007)^{42}$. Targeted stool samples analysis identified that patients following a low FODMAP diet had a significantly lower abundance of Bifidobacterium adolescentis, Bifidobacterium longum, 
and Faecalibacterium prausnitzii than patients on control diet with no differences in relation to microbiome diversity and markers of inflammation ${ }^{43}$.

\subsubsection{Specific carbohydrate}

Specific carbohydrate diet (SCD) is a nutrition strategy that limits the consumption of certain carbohydrates. A retrospective paediatric study aimed to evaluate the potential effects of the SCD in patients with active UC. The mean PUCAI for patients with active UC decreased from a baseline of $28.3 \pm 10.3$ to $20.0 \pm 17.3$ at $4 \pm 2$ week, to $18.3 \pm$ 31.7 at 6 mo. Evidence shows that the SCD may be effective in decreasing disease activity and deserves further investigation and possible integration in the community but mechanisms involving the gut microbiome are lacking ${ }^{44}$.

\subsubsection{Anti-inflammatory diet}

The Anti-Inflammatory Diet (IBD-AID) is a potential dietary therapy for IBD patients, restricting the ingestion of certain carbohydrates aiming to reduce symptoms and gut healing. A study recruiting forty patients with IBD were consecutively offered the IBDAID to help treat their disease and were retrospectively reviewed. Of those forty adult patients, eleven were included in the final analysis and underwent further medical record review. After following the IBD-AID for at least four weeks, all patients were able to discontinue at least one of their prior IBD medications, and all patients had improvement of their symptoms, including reduced bowel frequency suggesting a potentially beneficial effect of this dietary strategy in clinical outcomes ${ }^{45}$. Objective measures of inflammation, such as faecal calprotectin or endoscopy, were not assessed. The impact of this diet on the gut microbiome is still not fully elucidated. 


\subsubsection{High fibre}

Fibre ingestion can shape the structure of the gut microbiome and can contribute to colonic homeostasis, intestinal integrity, thus leading to a lower disease risk ${ }^{46}$. Resistant starch and pectin increase the relative abundance of butyrate-producing bacteria while reduction of dietary fibre consumptions is associated with a decrease in butyrateproducing bacteria such as Faecalibacterium, as well as an increase in mucus-eroding microbiota such as Akkermansia muciniphila and Bacteroides caccae. Dietary fibre is fermented in the colon and provide energy substrates for the colonocytes. Importantly, healthy subjects supplemented with fructooligosaccharides and galactooligosaccharides exhibit an increased abundance of Bifidobacteria and Lactobacilli ${ }^{47}$.

\subsubsection{Mediterranean diet}

The Mediterranean diet (Md) is largely known by its inflammatory characteristics and cardiovascular benefits. A prospective study aimed to identify the impact of Md on the nutritional state, liver steatosis, clinical disease. The study reported a significant reduction of malnutrition-related parameters and liver steatosis was observed in UC patients after short-term dietary intervention ${ }^{48}$. Specifically they noticed that after 6 months of a Mediterranean diet fewer patients with UC had active disease (14 of 59 [23.7\%] at start of trial vs 4 of 59 [6.8\%] at 6 months following diet, $P=0.004$ ) and furthermore, this study highlighted that a meditteranean diet was associated with an increase in quality of life ${ }^{48}$. It has been suggested that a Mediterranean diet might be associated with modulation of the gut mictobiome by increasing short/branch chained fatty acid production and lowering production of secondary bile acids, $p$ cresols, ethanol and carbon dioxide ${ }^{49}$. This was shown to be associated with a reduction in fragility but as yet mechanisms in ulcerative colitis has yet to be elucidated ${ }^{49}$.

\subsubsection{Gluten-free diet}


Gluten consists of proteins that are partially resistant to proteolytic digestion being a major dietary component in wheat, rye, and barley. Non-celiac gluten sensitivity and the associated use of a gluten-free diet (GFD) has been used as a dietary strategy to better control IBS symptoms and recently as a possible comprehensive tool to manage inflammatory bowel disease (IBD). In a study trying to analyse the effect of a GFD on gut inflammation, $65.6 \%$ of patients described an improvement of their gastrointestinal symptoms and $38.3 \%$ reported fewer or less severe IBD flares.(74). As of yet, it is unclear how a GFD affects the gut microbiome and therefore further prospective studies into mechanisms of gluten sensitivity in IBD are warranted ${ }^{50}$.

\subsubsection{Omega 3}

Clinical studies show that omega-3 fatty acids may have a possible role in the treatment of IBD. Deficiency in essential fatty acids is commonly seen in IBD patients, and omega3 fatty acids supplements may benefit patients through the inhibition of natural cytotoxicity (by changing arachidonic acid metabolites) and/or improving oxidative stress $^{51}$. In a cohort of heathy middle-aged and elderly women, both total omega-3 and DHA serum levels were significantly correlated with microbiome alpha-diversity after adjusting for confounders. Some of the associations with gut bacterial operational taxonomic unit appear to be mediated by the abundance of the faecal metabolite $\mathrm{N}$ carbamylglutamate. These data suggests link between omega-3 circulating levels/intake and microbiome composition independent of dietary fibre intake, particularly with bacteria of the Lachnospiraceae family ${ }^{52}$. More studies assessing the effect of omega-3 supplementation on the microbiota of IBD patients are required.

\section{$\underline{\text { 2.6.9 Curcumin }}$}

Polyphenols which constitute the active substances found in many plants, seem to have positive effects in the management of IBD via down-regulation of inflammatory cytokines and enzymes, enhancing antioxidant defence, and suppressing inflammatory 
pathways and the cellular signalling mechanisms ${ }^{53}$. Their specific role on the microbiome and ulcerative colitis remain unclear.

\subsection{Prebiotic studies in pouchitis}

Welters et al evaluated the effects of enteral inulin on ileo-anal pouch functioning by studying epithelial gene expression, cell turnover, and mucosal morphology. Twenty patients were given $24 \mathrm{~g}$ of inulin daily for three weeks, then a four-week wash-out period, and a placebo for three weeks. Inulin supplementation did not significantly alter pouch mucosal functioning because neither epithelial homeostasis nor epithelial gene expression was significantly altered; however, the author concluded that enteric supplementation with $24 \mathrm{~g} /$ day of inulin led to a decrease of inflammation-associated factors, with an increase in butyrate production, decrease of secondary bile acids and significant decrease in the endoscopic and histologic pouch disease activity index score $^{54}$. This has yet to be linked to the role in the microbiome but could provide some interesting mechanistics as to the importance of prebiotics in pouch integrity.

\subsection{Probiotic studies in pouchitis}

In a meta-analysis of the 4 RCTs, it was shown that probiotics have no effect on maintenance of remission in pouchitis. However, when exploring individual studies, it has been demonstrated that $6 \mathrm{~g} /$ day of a multistrain probiotic containing a combination of lactic acid bacteria, streptococci and bifidobacteria can help maintain remission ${ }^{55}$ and prevent acute pouchitis ${ }^{56,57}$. There is a single small trial suggestive that probiotics may be effective for acute pouchitis ${ }^{58}$ but this was not replicated in a randomised controlled trial $^{59}$ and hence its position in acute pouchitis remains uncertain.

When exploring some of the microbial mechanisms that changed during probiotic treatment of the pouch, Gionchetti et al. noted that faecal concentration of lactobacilli, bifidobacteria, and $S$. thermophilus increased significantly from baseline levels only in the group receiving the multistrain probiotic containing a combination of lactic acid 
bacteria, streptococci and bifidobacteria $(P<0.01)^{56}$. The same group in a follow-up study found that the patients that benefited from the multistrain probiotic were associated with faecal colonization with probiotics ${ }^{58}$. In the other probiotic study reporting on changes in the gut microbiome, Kuisima et al highlighted that a trial of Lactobacillus GG supplementation (10 LGG, 10 placebo) for 3 months changed the pouch microbiota, but was ineffective as primary therapy for a clinical or endoscopic response $^{60}$.

\subsection{Antibiotic studies in pouchitis}

Antibiotics remain the mainstay treatment for both acute and chronic pouchitis. In a meta-analysis it was highlighted that antibiotics could achieve remission in nearly three quarters of cases ${ }^{61}$. However, these are based on a number of small randomized controlled trials and observational studies. When exploring the microbial changes that occur following antibiotics, the evidence is very heterogenous, meaning consistent signals are not yet found ${ }^{62}$. Furthermore, the mechanisms that underpin the microbial changes that are responsible for the beneficial effect remains poorly understood. In one of the few mechanistic studies exploring the role of antibiotics and pouchitis, it was highlighted that antibiotics lead to an antibiotic-resistant microbiome with low virulence which helps to maintain remission ${ }^{63}$.

\subsection{FMT in pouchitis}

There have been a number of small studies now that have explored the potential of faecal microbiota transplantation for chronic pouchitis. Overall, a meta-analysis highlighted that as yet there was a lack of evidence for its effectiveness in the treatment of chronic pouchitis ${ }^{64}$. The problem with many of these studies is the heterogeneity in study design, the methodology of delivering the faecal transplant, the relatively small number of patients and the variability of diagnosis of pouchitis. Interpretation of these studies remains challenging, especially understanding mechanisms that may underpin therapeutic success. 


\section{$\underline{4.0 \text { Interventional Dietary studies in pouchitis }}$}

A longitudinal cohort study followed 172 patients within the first year after IPAA surgery investigating who developed pouchitis. There was a greater risk of pouchitis at 1 year in 13 patients with low fruit intake [ $30.8 \%$ for $<1.45$ servings fruit per day] compared with 26 patients with higher fruit intake [3.8\% for $>1.45$ servings fruit per day]. Additionally, higher fruit consumption correlated with increased microbial diversity and with higher abundance of various bacterial genera including Lachnospira, Lactobacillus, Faecalibacterium and Ruminococcus ${ }^{65}$.

Croagh et al evaluated the contribution of poorly absorbed short-chain carbohydrates (FODMAPs) in the diet to the pouch behaviour. Five of seven patients studied retrospectively improved stool frequency (from median 8 to 4 per day; $P=0.02$ ), this being sustained over 0.5-3 years of follow-up. Overall, none of eight patients who had pouchitis improved, however microbial mechanisms that underpin the efficacy is poorly understood 6 .

A study assessing patients' adherence to a Mediterranean diet included 153 UC pouch patients who responded to a 106-item FFQ. They noted that good adherence to Mediterranean diet at baseline was associated with a lower chance of developing pouchitis compared to poor adherence ( $26 \%$ vs $45 \%)$. However, the mechanisms that impact the gut microbiome are currently unknown ${ }^{67}$.

McLaughlin et al studied the impact of exclusive elemental diet on the gastrointestinal microbiota and symptoms in patients with chronic pouchitis. In their case series, 7 patients with pouchitis following IPAA for UC were treated with exclusive elemental diet therapy for 4 weeks. The median stool frequency significantly decreased from 12 to 6 per day. However, there was no significant difference in quality-of-life scores or pouch disease activity index before and after treatment. There were no significant changes in the concentration of bacteria after treatment. There was a trend towards an increase in the concentration of Clostridium coccoides and Eubacterium rectale. The authors 
concluded that elemental diet therapy appeared to improve the symptoms of pouchitis in some patients but are not an effective strategy for inducing remission ${ }^{68}$.

\subsection{Conclusion}

Through the modulation of the gut microbiome, we have a chance to personalize medicine, tackling inflammatory disorders by targeting microbes involved in the pathogenesis of disease.

\section{$\underline{\text { References }}$}

1. Vieira-Silva, S. et al. Species-function relationships shape ecological properties of the human gut microbiome. Nat. Microbiol. 1, 16088 (2016).

2. Carmen Collado, M. et al. Factors influencing gastrointestinal tract and microbiota immune interaction in preterm infants. (2015). doi:10.1038/pr.2015.54 
3. Pasvol, T. J. et al. Incidence and prevalence of inflammatory bowel disease in UK primary care: a population-based cohort study. BMJ Open 10, e036584 (2020).

4. Segal, J. P. et al. The application of omics techniques to understand the role of the gut microbiota in inflammatory bowel disease. Therap. Adv. Gastroenterol. 12, 175628481882225 (2019).

5. Lobionda, S., Sittipo, P., Kwon, H. Y. \& Lee, Y. K. The Role of Gut Microbiota in Intestinal Inflammation with Respect to Diet and Extrinsic Stressors.

Microorganisms 7, (2019).

6. Paone, P. \& Cani, P. D. Mucus barrier, mucins and gut microbiota: the expected slimy partners? Gut 69, 2232-2243 (2020).

7. Schnorr, S. L. et al. Gut microbiome of the Hadza hunter-gatherers. Nat. Commun. 5, 3654 (2014).

8. Sartor, R. B. Microbial influences in inflammatory bowel diseases. Gastroenterology 134, 577-594 (2008).

9. Komanduri, S., Gillevet, P. M., Sikaroodi, M., Mutlu, E. \& Keshavarzian, A. Dysbiosis in Pouchitis: Evidence of Unique Microfloral Patterns in Pouch Inflammation. Clin. Gastroenterol. Hepatol. 5, 352-360 (2007).

10. Sartor, R. B. Microbial Influences in Inflammatory Bowel Diseases. Gastroenterology 134, 577-594 (2008).

11. Machiels, K. et al. Specific members of the predominant gut microbiota predict pouchitis following colectomy and IPAA in UC. Gut 66, 79-88 (2017).

12. Gibson, G. R. et al. Expert consensus document: The International Scientific Association for Probiotics and Prebiotics (ISAPP) consensus statement on the definition and scope of prebiotics. Nat. Rev. Gastroenterol. Hepatol. 14, 491-502 (2017).

13. Camuesco, D. et al. Preventative Effects of Lactulose in the Trinitrobenzenesulphonic Acid Model of Rat Colitis. Inflamm. Bowel Dis. 11, 265271 (2005).

14. Hafer, A. et al. Effect of oral lactulose on clinical and immunohistochemical parameters in patients with inflammatory bowel disease: a pilot study. BMC Gastroenterol. 7, 36 (2007). 
15. CASELLAS, F. et al. Oral oligofructose-enriched inulin supplementation in acute ulcerative colitis is well tolerated and associated with lowered faecal calprotectin. Aliment. Pharmacol. Ther. 25, 1061-1067 (2007).

16. Valcheva, R. et al. Inulin-type fructans improve active ulcerative colitis associated with microbiota changes and increased short-chain fatty acids levels. Gut Microbes 10, 334-357 (2019).

17. Derwa, Y., Gracie, D. J., Hamlin, P. J. \& Ford, A. C. Systematic review with metaanalysis: the efficacy of probiotics in inflammatory bowel disease. Aliment. Pharmacol. Ther. 46, 389-400 (2017).

18. Astó, E., Méndez, I., Audivert, S., Farran-Codina, A. \& Espadaler, J. The Efficacy of Probiotics, Prebiotic Inulin-Type Fructans, and Synbiotics in Human Ulcerative Colitis: A Systematic Review and Meta-Analysis. Nutrients 11, (2019).

19. Kato, K. et al. Randomized placebo-controlled trial assessing the effect of bifidobacteria-fermented milk on active ulcerative colitis. Aliment. Pharmacol. Ther. 20, 1133-1141 (2004).

20. Yoshimatsu, Y. et al. Effectiveness of probiotic therapy for the prevention of relapse in patients with inactive ulcerative colitis. World J. Gastroenterol. 21, 5985 (2015).

21. Matsuoka, K. et al. Efficacy of Bifidobacterium breve Fermented Milk in Maintaining Remission of Ulcerative Colitis. Dig. Dis. Sci. 63, 1910 (2018).

22. Furrie, E. et al. Synbiotic therapy (Bifidobacterium longum/Synergy 1 ) initiates resolution of inflammation in patients with active ulcerative colitis: a randomised controlled pilot trial. Gut 54, 242 (2005).

23. Khan, K. J. et al. Antibiotic Therapy in Inflammatory Bowel Disease: A Systematic Review and Meta-Analysis. Am. J. Gastroenterol. 106, 661-673 (2011).

24. Singh, S., Allegretti, J. R., Siddique, S. M. \& Terdiman, J. P. American Gastroenterological Association Technical Review on the Management of Moderate to Severe Ulcerative Colitis. Gastroenterology 158, 1465 (2020).

25. Kennedy, N. A. et al. British Society of Gastroenterology guidance for management of inflammatory bowel disease during the COVID-19 pandemic. Gut (2020). doi:10.1136/GUTJNL-2020-321244 
26. Moayyedi, P. et al. Fecal Microbiota Transplantation Induces Remission in Patients With Active Ulcerative Colitis in a Randomized Controlled Trial. Gastroenterology 149, 102-109.e6 (2015).

27. Rossen, N. G. et al. Findings From a Randomized Controlled Trial of Fecal Transplantation for Patients With Ulcerative Colitis. Gastroenterology 149, 110 118.e4 (2015).

28. Paramsothy, S. et al. Multidonor intensive faecal microbiota transplantation for active ulcerative colitis: a randomised placebo-controlled trial. Lancet 389, 12181228 (2017).

29. Costello, S. P. et al. Effect of Fecal Microbiota Transplantation on 8-Week Remission in Patients With Ulcerative Colitis. JAMA 321, 156 (2019).

30. Cold, F. et al. Multidonor FMT capsules improve symptoms and decrease fecal calprotectin in ulcerative colitis patients while treated - an open-label pilot study. Scand. J. Gastroenterol. 54, 289-296 (2019).

31. Mańkowska-Wierzbicka, D. et al. The Effectiveness of Multi-Session FMT Treatment in Active Ulcerative Colitis Patients: A Pilot Study. Biomedicines 8, (2020).

32. Sood, A. et al. Role of Faecal Microbiota Transplantation for Maintenance of Remission in Patients With Ulcerative Colitis: A Pilot Study. J. Crohn's Colitis (2019). doi:10.1093/ecco-jcc/jjz060

33. Quraishi, M. N. N. et al. STOP-Colitis pilot trial protocol: a prospective, open-label, randomised pilot study to assess two possible routes of faecal microbiota transplant delivery in patients with ulcerative colitis. BMJ Open 9, e030659 (2019).

34. Costello, S. P. et al. Systematic review with meta-analysis: faecal microbiota transplantation for the induction of remission for active ulcerative colitis. Aliment. Pharmacol. Ther. 46, 213-224 (2017).

35. Paramsothy, S. et al. Specific Bacteria and Metabolites Associated With Response to Fecal Microbiota Transplantation in Patients With Ulcerative Colitis. Gastroenterology 156, 1440-1454.e2 (2019).

36. Conceição-Neto, N. et al. DOP080 Low viral richness at baseline in ulcerative 
ulcerative colitis associated with faecal microbiota transplantation success. J. Crohn's Colitis 11, S73-S74 (2017).

37. Leonardi, I. et al. Fungal Trans-kingdom Dynamics Linked to Responsiveness to Fecal Microbiota Transplantation (FMT) Therapy in Ulcerative Colitis. Cell Host Microbe 27, 823-829.e3 (2020).

38. Li, F., Liu, X., Wang, W. \& Zhang, D. Consumption of vegetables and fruit and the risk of inflammatory bowel disease. Eur. J. Gastroenterol. Hepatol. 27, 623-630 (2015).

39. Ananthakrishnan, A. N. et al. Long-term intake of dietary fat and risk of ulcerative colitis and Crohn's disease. Gut 63, 776-784 (2014).

40. Devkota, S. et al. Dietary-fat-induced taurocholic acid promotes pathobiont expansion and colitis in II10-/- mice. Nature 487, 104-108 (2012).

41. Staudacher, H. M., Irving, P. M., Lomer, M. C. E. \& Whelan, K. Mechanisms and efficacy of dietary FODMAP restriction in IBS. Nat. Rev. Gastroenterol. Hepatol. 11, 256-266 (2014).

42. Cox, S. R. et al. Effects of Low FODMAP Diet on Symptoms, Fecal Microbiome, and Markers of Inflammation in Patients With Quiescent Inflammatory Bowel Disease in a Randomized Trial. Gastroenterology 158, 176-188.e7 (2020).

43. Prince, A. C. et al. Fermentable Carbohydrate Restriction (Low FODMAP Diet) in Clinical Practice Improves Functional Gastrointestinal Symptoms in Patients with Inflammatory Bowel Disease. Inflamm. Bowel Dis. 22, 1129-1136 (2016).

44. Obih, C. et al. Specific carbohydrate diet for pediatric inflammatory bowel disease in clinical practice within an academic IBD center. Nutrition 32, 418-425 (2016).

45. Olendzki, B. C. et al. An anti-inflammatory diet as treatment for inflammatory bowel disease: a case series report. Nutr. J. 13, 5 (2014).

46. Flint, H. J., Scott, K. P., Louis, P. \& Duncan, S. H. The role of the gut microbiota in nutrition and health. Nat. Rev. Gastroenterol. Hepatol. 9, 577-589 (2012).

47. So, D. et al. Dietary fiber intervention on gut microbiota composition in healthy adults: a systematic review and meta-analysis. Am. J. Clin. Nutr. 107, 965-983 (2018).

48. Chicco, F. et al. Multidimensional Impact of Mediterranean Diet on IBD Patients. 
Inflamm. Bowel Dis. 27, 1-9 (2021).

49. Ghosh, T. S. et al. Mediterranean diet intervention alters the gut microbiome in older people reducing frailty and improving health status: the NU-AGE 1-year dietary intervention across five European countries. Gut 69, 1218-1228 (2020).

50. Herfarth, H. H., Martin, C. F., Sandler, R. S., Kappelman, M. D. \& Long, M. D. Prevalence of a gluten free diet and improvement of clinical symptoms in patients with inflammatory bowel diseases. Inflamm. Bowel Dis. 20, 1194 (2014).

51. Barbalho, S. M., Goulart, R. de A., Quesada, K., Bechara, M. D. \& Carvalho, A. de C. A. de. Inflammatory bowel disease: can omega-3 fatty acids really help? Ann. Gastroenterol. Q. Publ. Hell. Soc. Gastroenterol. 29, 37 (2016).

52. Menni, C. et al. Omega-3 fatty acids correlate with gut microbiome diversity and production of $\mathrm{N}$-carbamylglutamate in middle aged and elderly women. Sci. Rep. 7, 11079 (2017).

53. Lu, Y. et al. Dietary Polyphenols in the Aetiology of Crohn's Disease and Ulcerative Colitis-A Multicenter European Prospective Cohort Study (EPIC). Inflamm. Bowel Dis. 23, 2072-2082 (2017).

54. Welters, C. F. M. et al. Effect of dietary inulin supplementation on inflammation of pouch mucosa in patients with an ileal pouch-anal anastomosis. Dis. Colon Rectum 45, 621-7 (2002).

55. Mimura, T. et al. Once daily high dose probiotic therapy (VSL\#3) for maintaining remission in recurrent or refractory pouchitis. Gut 53, 108 (2004).

56. Gionchetti, P. et al. Prophylaxis of pouchitis onset with probiotic therapy: A double-blind, placebo-controlled trial. Gastroenterology 124, 1202-1209 (2003).

57. Gionchetti, P. et al. Oral bacteriotherapy as maintenance treatment in patients with chronic pouchitis: a double-blind, placebo-controlled trial. Gastroenterology 119, 305-9 (2000).

58. Gionchetti, P. et al. High-Dose Probiotics for the Treatment of Active Pouchitis. Dis. Colon Rectum 50, 2075-2084 (2007).

59. Bengtsson, J. et al. Effect of probiotics ( Lactobacillus plantarum 299 plus Bifidobacterium Cure21) in patients with poor ileal pouch function: a randomised controlled trial. Scand. J. Gastroenterol. 51, 1087-1092 (2016). 
60. Kuisma, J. et al. Effect of Lactobacillus rhamnosus GG on ileal pouch inflammation and microbial flora. Aliment. Pharmacol. Ther. 17, 509-15 (2003).

61. Segal, J. P. et al. Systematic review with meta-analysis: the management of chronic refractory pouchitis with an evidence-based treatment algorithm. Aliment. Pharmacol. Ther. 45, (2017).

62. Segal, J. P. et al. Systematic review: ileoanal pouch microbiota in health and disease. Aliment. Pharmacol. Ther. 47, (2018).

63. Dubinsky, V. et al. Predominantly Antibiotic-resistant Intestinal Microbiome Persists in Patients With Pouchitis Who Respond to Antibiotic Therapy. Gastroenterology 158, 610-624.e13 (2020).

64. Kayal, M., Lambin, T., Pinotti, R., Dubinsky, M. C. \& Grinspan, A. A Systematic Review of Fecal Microbiota Transplant for the Management of Pouchitis. Crohn's Colitis 360 2, (2020).

65. Godny, L. et al. Fruit Consumption is Associated with Alterations in Microbial Composition and Lower Rates of Pouchitis. J. Crohn's Colitis 13, 1265-1272 (2019).

66. Croagh, C., Shepherd, S. J., Berryman, M., Muir, J. G. \& Gibson, P. R. Pilot study on the effect of reducing dietary FODMAP intake on bowel function in patients without a colon. Inflamm. Bowel Dis. 13, 1522-1528 (2007).

67. Godny, L. et al. Adherence to the Mediterranean diet is associated with decreased fecal calprotectin in patients with ulcerative colitis after pouch surgery. Eur. J. Nutr. 59, 3183-3190 (2020).

68. McLaughlin, S. D. et al. Exclusive elemental diet impacts on the gastrointestinal microbiota and improves symptoms in patients with chronic pouchitis. J. Crohn's Colitis 7, 460-466 (2013). 